\title{
Early Histological Changes in Post-Transplant Encapsulating Peritoneal Sclerosis
}

\author{
Gioacchino Li Cavoli Antonio Amato Rosalia Mongiovì Onofrio Schillaci \\ Carlo Giammarresi Luisa Bono Rosa Turdo Camillo Carollo Carmela Zagarrigo \\ Franca Servillo Barbara Oliva Angelo Tralongo Flavia Caputo \\ Department of Nephrology Dialysis and Renal Transplant, Civico Hospital, Palermo, Italy
}

\section{Dear Editor,}

Peritoneal Dialysis (PD) is a cost-effective renal replacement therapy that offers patients the convenience of a homebased treatment, a flexible schedule and an increased perception of freedom; along with its low hemodynamic instability, PD often results in a better quality of life. When exposed to PD dialysis solutions, the peritoneal membrane undergoes some morphological changes: a thin layer of submesothelial fibrosis is often highlighted, with a thickness not exceeding a few hundred microns; a component of neoangiogenesis, not associated with significant vascular damage, is often demonstrable; calcifications are rare and so are the signs of a significant inflammatory state [1]. This condition identifies what is known as the Simple Peritoneal Sclerosis. A minority of patients on PD develops the Encapsulating Peritoneal Sclerosis (EPS), a rare complication of long-term $\mathrm{PD}$, which consists in a progressive inflammatory process involving both visceral and parietal peritoneum, leading to encapsulation of the adhered intestinal tract. EPS features marked fibrosis, acute and chronic inflammation, widespread calcification and vascular thickening. Some authors think that Simple Peritoneal Sclerosis and EPS are the extremes of the continuous spectrum of a single disease related to $\mathrm{PD}$ biocompatibility. EPS may become clinically apparent when patients are on PD (classical EPS) or after undergoing kidney transplantation (post-transplantation EPS) [2]. This presentation of EPS seems to occur shortly after kidney transplantation in former PD patients. The critical phase for post-transplantation EPS is during the first year after transplantation. The pathophysiology of EPS is still unknown. We report our experience. A 61-year-old patient, for 10 years on PD, underwent kidney transplantation from deceased kidney donors; a few days afterwards, she started to complain about a constellation of dyspeptic digestive symp- toms: nausea, vomiting, anorexia, early satiety, constipation, abdominal fullness and pain. The peritoneal catheter was removed and biopsy of peritoneal membrane was carried out. The peritoneal histological examination highlighted signs of peritoneal sclerosis: thickening of peritoneal membrane (>600 micrometers), progressive fibrosis from the submesothelial layer towards the inside layer, marked thickening of middle vascular wall and mesothelial denudation. We did not find signs of active or chronic inflammation or peritoneal calcifications (Fig. 1). The Abdomen contrast-CT showed adherent and conglomerate intestinal loops (cocooning; Fig. 2). Based on symptomatology and radiological and histological findings, we diagnosed a recent onset of EPS. Due the profibrotic effects of calcineurin inhibitors, we stopped treatment with tacrolimus. Because of the evidence supporting protection against the development of EPS exerted by inhib-
KARGER

(C) 2018 S. Karger AG, Basel

E-Mail karger@karger.com

www.karger.com/bpu
Li Cavoli Gioacchino

Department of Nephrology Dialysis and

Renal Transplant, Civico Hospital

via Francesco Cilea 43, IT-90144 Palermo (Italy)

E-Mail gioacchinolicavoli@ @ibero.it 


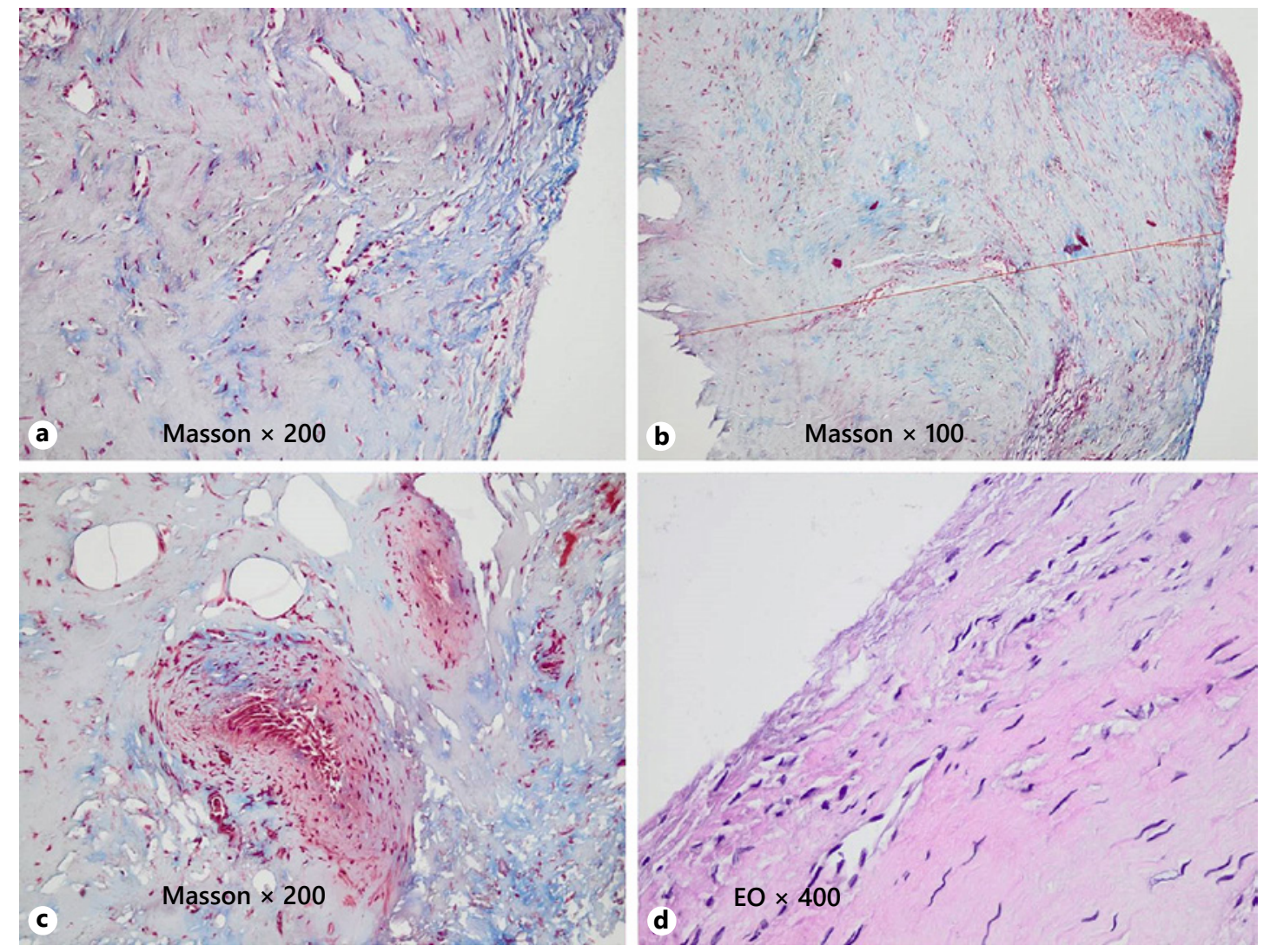

Fig. 1. Peritoneal histological examination: (a, b) the fibrous components of recent deposition, still rich in mucopolysaccharides, is in a pale color, while the more ancient fibrotic component, consisting almost exclusively of collagen, is highlighted in deeper blue. This staining highlights a recent beginning of the fibrotic process: blue is still poorly represented compared to the pale color. The thickness of the peritoneal membrane is increased (638 micronmeters). c Also in perivascular areas, the fibrosis spreads from the submesothelial layer towards the inside. $\mathbf{d}$ Marked thinning of the mesothelial layer.
Fig. 2. Abdomen contrast-CT: adherent and conglomerate intestinal loops (red arrow).

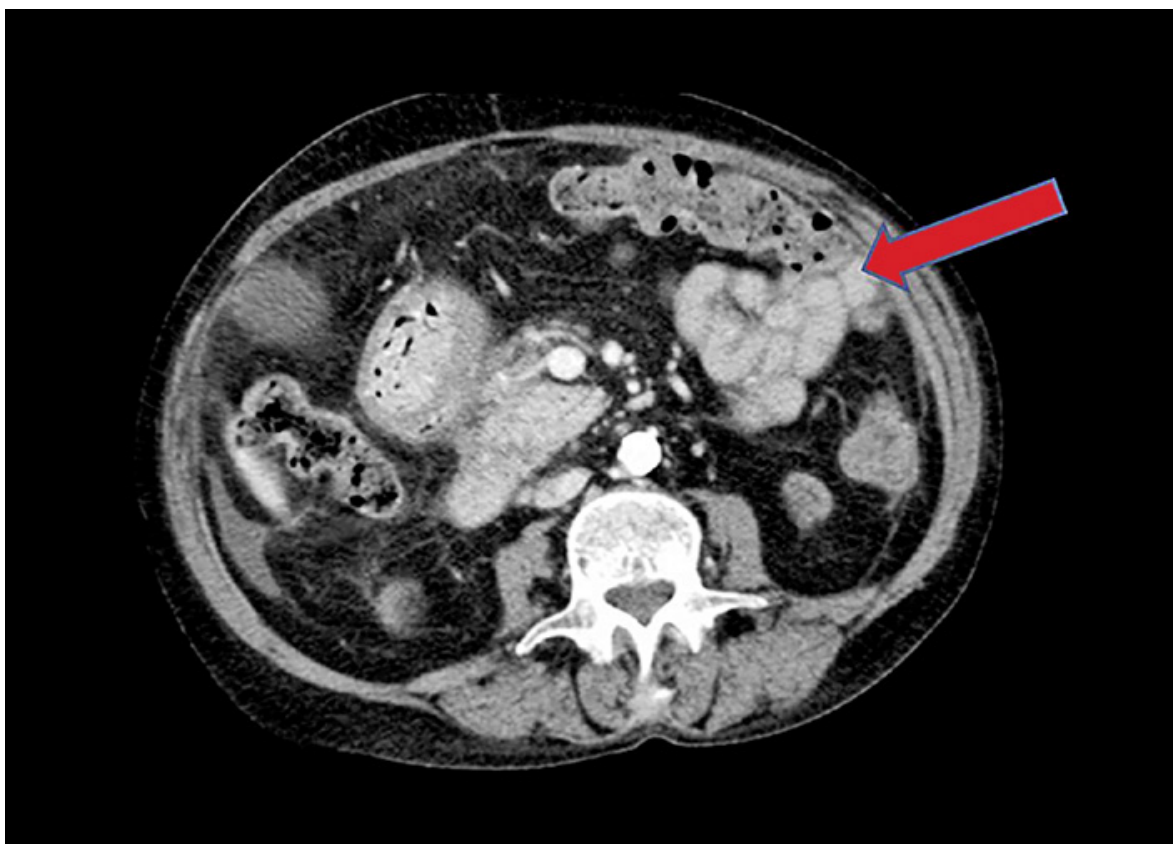


itors of the mammalian target of rapamycin, we started therapy with everolimus. Besides a transient increase in steroid therapy, we started therapy with tamoxifen. In the following 6 months of follow-up, the patient's symptoms ameliorated and her condition improved with regard to the bowel sub-occlusive crisis. Many factors have been incriminated in pathogenesis of EPS, but they do not explain all risk conditions. The risk of EPS increases with longer time on PD. Probably unidentified factors make some patients more susceptible to developing EPS. Early referral to transplantation may contribute significantly to preventing EPS in PD patients [3].

\section{References}

1 Garosi G, Mancianti N, Corciulo R, La Milia V, Virga G: Encapsulating peritoneal sclerosis. J Nephrol 2013;26:177-187.

\section{Acknowledgements}

The authors would like to express special thanks to Prof. Guido Garosi, Nephrology and Dialysis Unit, University Hospital of Siena, Siena - Italy.

\section{Disclosure Statement}

The authors declare that they have no conflicts of interest to disclose.
2 Williams JD, Craig KJ, Topley N, Von Ruhland C, Fallon M, Newman GR, et al; Peritoneal Biopsy Study Group: Morphologic changes in the peritoneal membrane of patients with renal disease. J Am Soc Nephrol 2002;13:470-479.
3 Li Cavoli G, Tralongo A, Schillaci O, Ferrantelli A, Li Cavoli TV, et al: A case of acute EPS with local cocoon formation in a patient on peritoneal dialysis. CEN Case Rep 2015;4: 243-245. 